$16^{\text {th }}$ International Conference on

AEROSPACE SCIENCES \& AVIATION TECHNOLOGY,

ASAT - 16 - May 26 - 28, 2015, E-Mail: asat@ mtc.edu.eg

Military Technical College, Kobry Elkobbah, Cairo, Egypt

Tel : +(202) 24025292 - 24036138, Fax: +(202) 22621908

\title{
Numerical Investigation of Wind Tunnel Wall Effects on a Supersonic Finned Missile
}

\author{
Loai A. Elmahdy ${ }^{*}$, M.Y.M Ahmed*, O.K. Mahmoud ${ }^{*}$ and O.E. Abdel Hamid*
}

\begin{abstract}
One major aspect of wind tunnel measurements is the influence of test section walls on the measured data. In this paper, a numerical investigation of the supersonic flow around a conventional missile configuration is conducted. Wall effects are addressed by comparing two computational domains, unbounded and bounded.

The calculated aerodynamic coefficients are compared with own corresponding measured values. The results show a good agreement between the measured parameters and those derived from the bounded computational domain. The impact of the walls on the flow field structure is investigated by comparing the bounded and unbounded numerical simulations.
\end{abstract}

Keywords: missile aerodynamics, CFD, wall effect

\section{Nomenclature:}

$\mathrm{C}_{\mathrm{A}}$ axial force coefficient.

$\mathrm{C}_{\mathrm{df}}$ fore body drag coefficient.

$\mathrm{C}_{\mathrm{m}} \quad$ pitching moment coefficient.

$\mathrm{C}_{\mathrm{N}} \quad$ normal force coefficient.

$\mathrm{X}_{\mathrm{cp}} \quad$ normalized center of pressure position.

\section{Introduction}

Generally, there are three possible methods to estimate aerodynamic coefficients for a flying body namely, experimental, computational, and analytical. Experimental methods in which the aerodynamic coefficients are measured on an aerodynamically similar model in a wind tunnel, should be the closest to real flights. However, they are expensive, time consuming, and need measurement facilities that may not be available. Moreover, some real flight conditions may not be replicable in wind tunnels. In the Computational methods, based on computational fluid dynamics, CFD approach, the flow around the body is simulated by solving the governing equations of the flow numerically in the computational domain around the body. Once the simulation is properly set, the computation cost and time can be more economic compared with experimental methods. The processes for the preparation of CFD simulations can be complicated however, they are indeed more flexible compared with experimental measurements. The advantage of this method is the ability for visualizing flow features. Finally in the analytical method, simplified, closed form, and empirical equations are used. These equations are the simplest and least expensive and time consuming. However, they are basically suitable for simple flight problems in terms of body geometry and flight conditions. They yield poor results for high flight speeds or incidences.

\footnotetext{
*Egyptian armed forces
} 
Wind tunnel measurements should be as close as possible to real flight. However, it has been shown that these measurements are subject to various types of errors. They are listed briefly as[1]:

(1) Solid Blockage: the free stream velocity around the model increases due to the confinement of the flow.

(2) Streamline curvature: The normal curvature of the flow around a model is straightened by the tunnel walls. For subsonic airfoils in the tunnel, more lift and moment will be measured at a given angle of attack.

(3) Wake blockage: The blockage due to the wake of the model. This may influence the upstream flow especially in subsonic conditions

(4) Scaling error: the scaled model gives values that are different from those of the full scale.

(5) Shock reflections: the source of error is witnessed in transonic and supersonic free stream conditions. Shock waves that are created around the model are reflected on the tunnel walls. The reflected waves may impinge the model causing changes in the measured parameters.

(6) Horizontal buoyancy: the boundary layer on the test section walls has the effect of reducing the flow area of the free stream.

All the above mentioned errors are commonly referred as "wall interference errors".

Estimating and correcting the wind tunnel wall effects has drawn the attention of researchers for decades. For example,

In 1944 Allen and Vincbnti[2] derived theoretical tunnel-wall corrections for an airfoil of finite thickness and camber in a two dimensional flow wind tunnel. In their study, they considered the effect of the wake of the airfoil and of the compressibility of the fluid with the restriction that the chord of the wing is small compared with the tunnel height. They presented correction equations for the different parameters measured in the wind tunnel at low Mach numbers, however it has only limited applications at higher Mach numbers.

Thom and Klanfer[3] studied the effect of the presence of walls on an airfoil in a subsonic flow. They found that: the walls of the wind tunnel increase the surface velocity on the airfoil. To get an accurate results and a closer simulation for the real flow they suggested using a thinner airfoil, which leads to another wind tunnel error namely, scaling error.

Maskell[4] studied the effect of blockage on a wing and presented empirical correction equations depending on the dynamic pressure and the value of base pressure coefficient which showed good results with the experimental work.

Awbi[5] experimentally investigated the effect of wall constraint on rectangular cross-section prism in a subsonic wind tunnel for different blockage and depth-to-width ratios of the test section. He showed that the effect of confinement of the flow on the mean surface pressure on the model was not uniform in the stream wise direction.

West and Apelt[6] studied the blockage effects on the base pressure and on the drag coefficients of circular cylinders using different blockage and aspect ratios. They found that for blockage ratios above $6 \%$ the pressure distribution differed from that with smaller ratios while the change in drag coefficient was small compared with that with no blockage. They also concluded that reduction in aspect ratio affects drag coefficient and base pressure coefficient which were similar to those associated with the increase in blockage ratio.

Duraisamy[7] studied the effect of wall interference on steady and oscillating airfoils in a subsonic wind tunnel using various theoretical methods, compressible, inviscid and viscous computations, and compared his work with experimental data. He presented a methodology 
that could be used to predict wall interference effects and to extrapolate linear and nonlinear wind tunnel data to free-air conditions.

The above-mentioned studies focused on bodies exposed to subsonic free stream conditions. In the open literature, the wind tunnel wall effects in the supersonic free-stream conditions were treated only recently. Ivanov and Streltsov[8] in 2014 introduced a new method to the boundary interference problem for subsonic and transonic wind tunnel which is based on the idea of using controlled boundary layer on the test section walls.

Doig[9] studied the aerodynamics of a projectile in proximity to a solid wall flying at Mach 2.4 at zero incidence using the computational fluid dynamics approach. Considering the spin of the projectile, he studied the effect of changing the distance from the wall. It was concluded that the proximity increased the drag and generated a normal force on the projectile for wall clearance less than one caliber. For values of wall clearance between 0.4 and 1 caliber, the pitching moment produced was nose-down, for lower clearances, a more distinct nose-up trend was produced.

Finally, Roychowdhury and Unnikrishnan[10] investigated the wall interference and solid blockage on a supersonic blunt model representing the payload of a launch vehicle. The simulation was axisymmetric (for zero angle of attack), at Mach numbers 1.5 and 3 for various blockage ratios. This approach implies cylindrical test section. It was found that when the blockage ratio is small, the detached shock extends to the tunnel wall reflecting back to the body various times which makes oscillations in the pressure distribution on the surface of the bod. The study presented recommendations for the blockage ratio and the model length to get smooth pressure distribution un-affected by the wall presence. However the study lacks for validation with experimental measurements.

The present paper aims to shed more light on the wall effects in supersonic wind tunnel measurements. The supersonic flow around a model of a finned missile at incidence is simulated numerically. The free stream Mach number varies from 1.5 to 4 at $4^{\circ}$ incidence angle.

To address the impact of test section walls, two 3-dimensional computational domains are investigated namely, open (unbounded) and closed (bounded with test section walls). The flow field features in both domains are explored and compared, and the overall aerodynamic properties are compared with own wind tunnel measurements.

The remainder of the paper is organized as follows, details of the case study are presented next followed by the simulation setup details. The main results are then discussed and paper finalizes with the key findings.

\section{Case study}

\subsection{Model}

The model investigated is a 1:16 scaled missile body configuration composed of conecylinder body, two outer conduits, and four fins. The model has a total length and caliber of $551.25 \mathrm{~mm}$ and $34 \mathrm{~mm}$, respectively. The sketch of the model configuration is shown in Fig. 1 


\subsection{5}
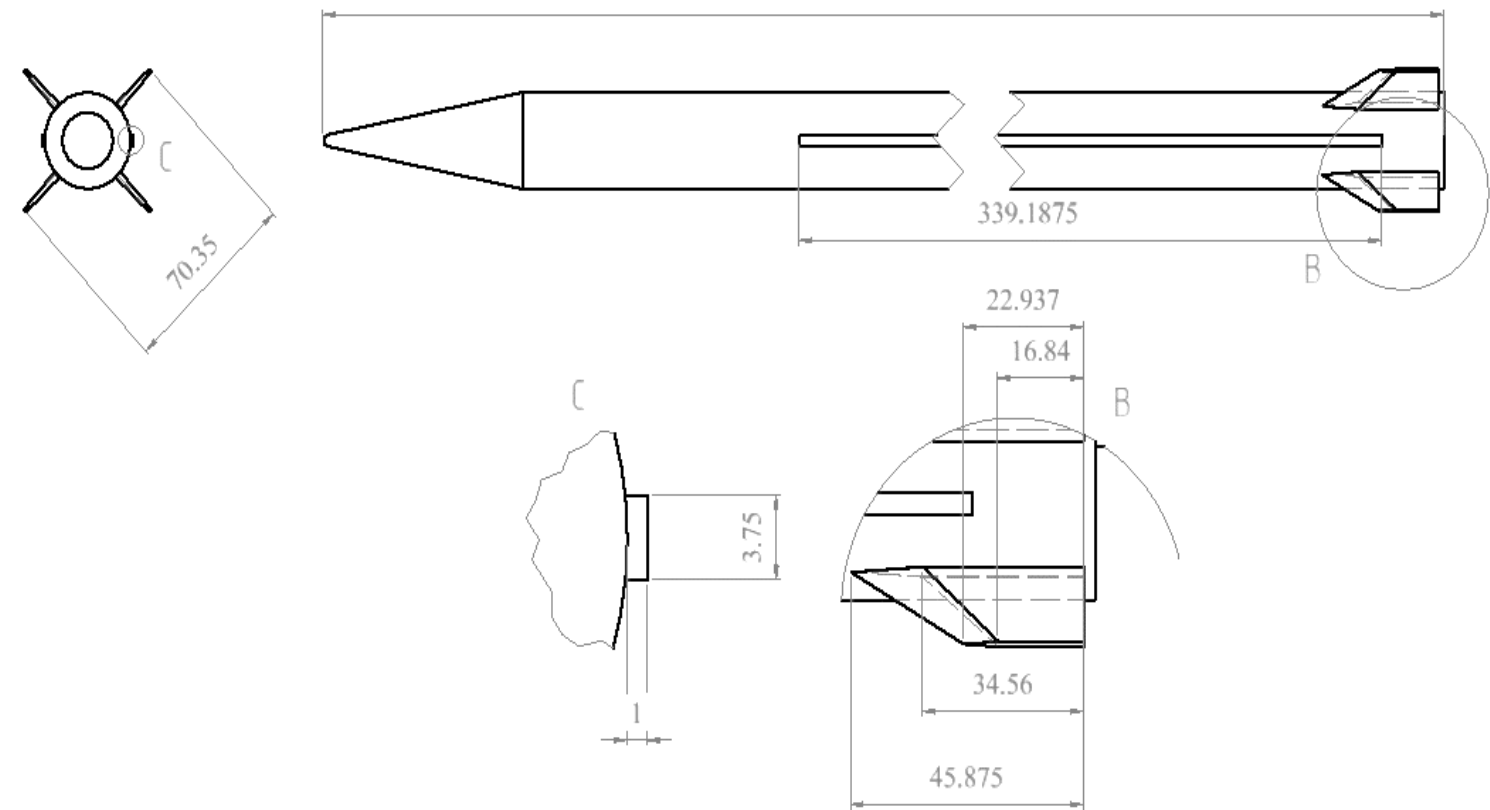

\section{Fig. 1 Full model configuration}

\subsection{Wind tunnel measurements}

The model is tested in a tri-sonic wind tunnel which test section dimensions are $0.6 \times 0.6 \mathrm{~m}^{2}$ and the length is $1.575 \mathrm{~m}$. The test conditions are variable from Mach 0.4 to 4.45 , the corresponding Reynolds number of the flow at the test section inlet varies from $8.7 \mathrm{e} 06$ to 26.5e6. A set of conventional attack angle mechanism is installed in the test section of the wind tunnel, which can change the attack angle in the range of $-15^{\circ} \sim 38^{\circ}$. The aerodynamic loads on the model are directly measured using a sting balance that is internally fit in the model base. Fig. 2 shows the model fitted inside the test section.
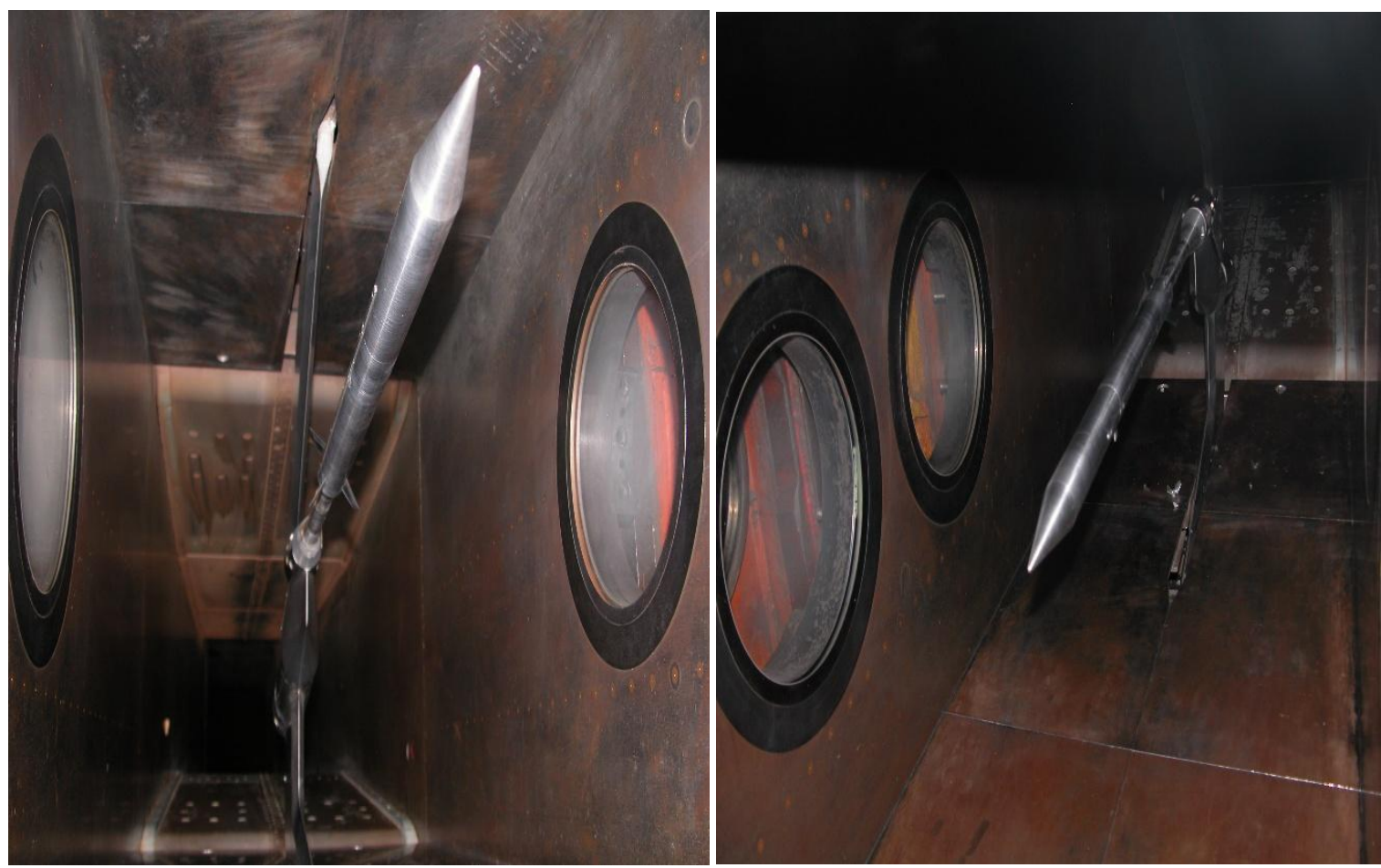

Fig. 2 model installation in the wind tunnel test section 
The accuracy of recorded data in measurements of the aerodynamic loads are listed in Table 1 .

Table 1 Recorded measurement errors for different parameters

\begin{tabular}{|c|c|c|}
\hline$\varepsilon_{\mathrm{CN}}$ & $\varepsilon_{\mathrm{CAF}}$ & $\varepsilon_{\mathrm{CAB}}$ \\
\hline \pm 0.0016 & \pm 0.0042 & \pm 0.0007 \\
\hline
\end{tabular}

Where

$\varepsilon_{\mathrm{CN}}$ is the error in measured normal force coefficient.

$\varepsilon_{\mathrm{CAF}}$ is the error in measured axial force coefficient on the forebody.

$\varepsilon_{\mathrm{CAB}}$ is the error in measured axial force coefficient on the base.

\subsection{Computational domain}

As stated earlier, CFD simulation is used to illustrate the effect of the presence of test section walls on the aerodynamic parameters of the model and the flow features around the model.

To do so, bounded and unbounded flow simulations are conducted in two different domains each of which should have the proper definitions of boundaries. Both domains are threedimensional. Since only the incidence angle is considered, the flow around the model is pitchplane symmetric. Thus, only half 3D domains are constructed.

The bounded domain is simply identical to the test section of the wind tunnel. The domain is defined by the dimensions of the test section, whereas the model is placed inclined in the test section orientation in the middle of the test section yielding the angle of attack of the model with respect to the flow through the test section. In Fig. 3 the bounded computational domain is illustrated. To replicate the wind tunnel experiments as closely as possible, the sting balance is added to the model. It is represented as cylindrical wall boundary that extends from the model base to the end of the domain.

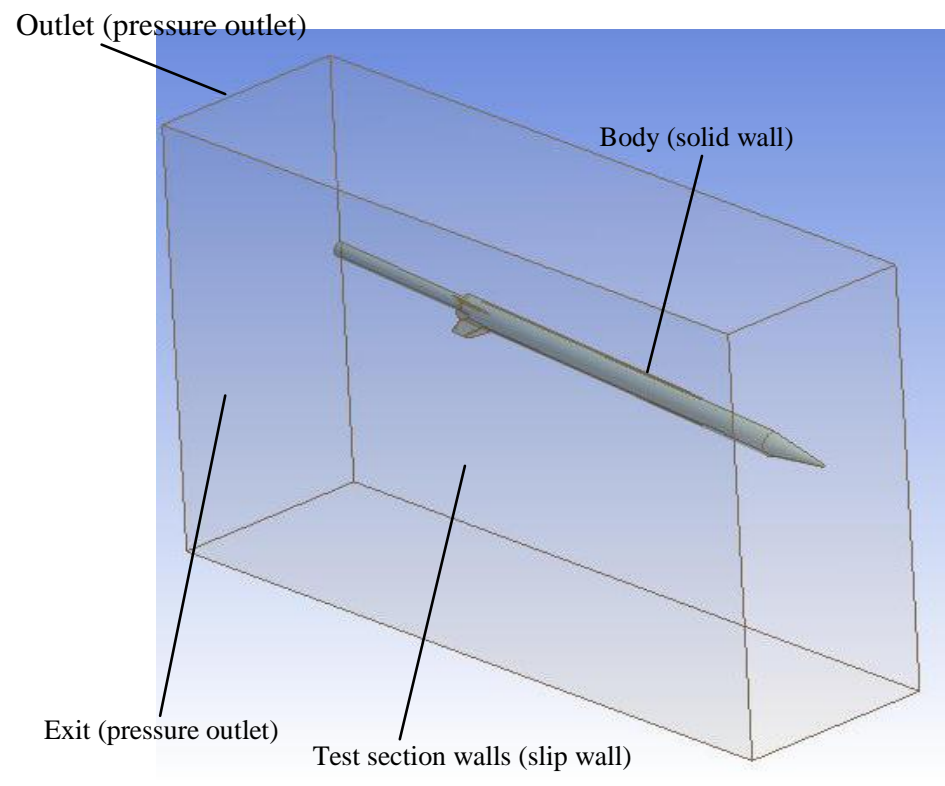

Fig. 3 Bounded computational domain

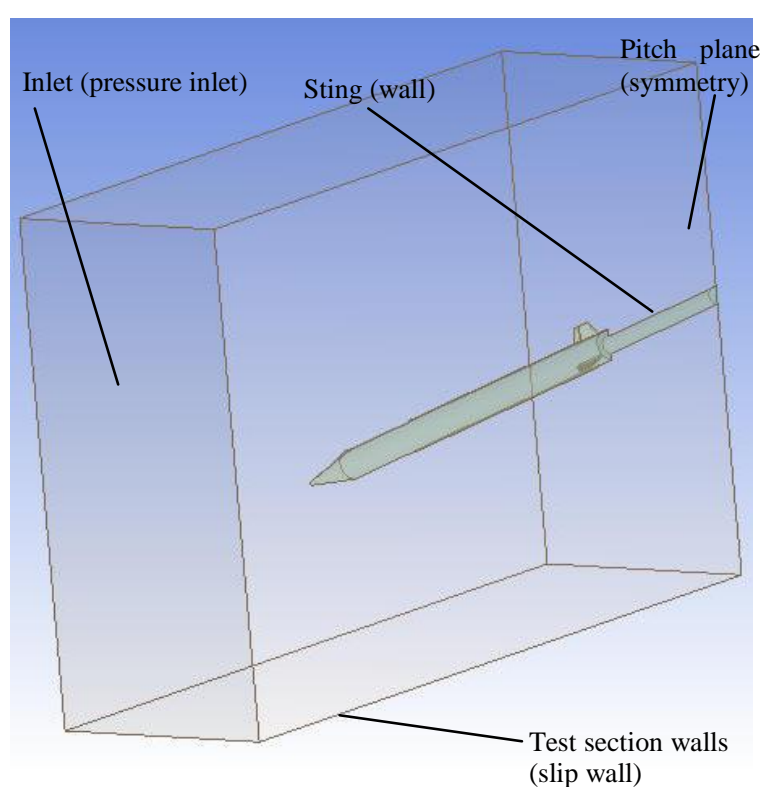

As shown in Fig. 3, the pitch plane is defined as symmetric boundary whereas the model surface is defined as solid wall with no-slip condition specified. All side walls of the test sections are represented as slip wall boundaries on which no boundary layer is created numerically. The inlet and exit of the test section are represented as pressure inlet and pressure outlet boundaries, respectively. 
In contrast, the unbounded domain is designed so that it captures all possible flow features that would happen around the model in the unbounded flight.

The unbounded domain has the form of a spherical domain in the middle of which the model is positioned. Domain extents are 4 calibers, 6 calibers, 20 calibers in the upstream, downstream, and the lateral directions, respectively. Such dimensions are found to adequately accommodate all flow features of the supersonic flow conditions in concern, in the unbounded domain, the model is drawn aligned with the coordinate system while the angle of attack is set by defining an inclined incoming free stream flow. Definitions of model surface, pitch plane, sting, and exit are similar to those in the bounded domain. The outer boundary of the domain is defined as pressure far field. Fig. 4 shows the unbounded computational domain.

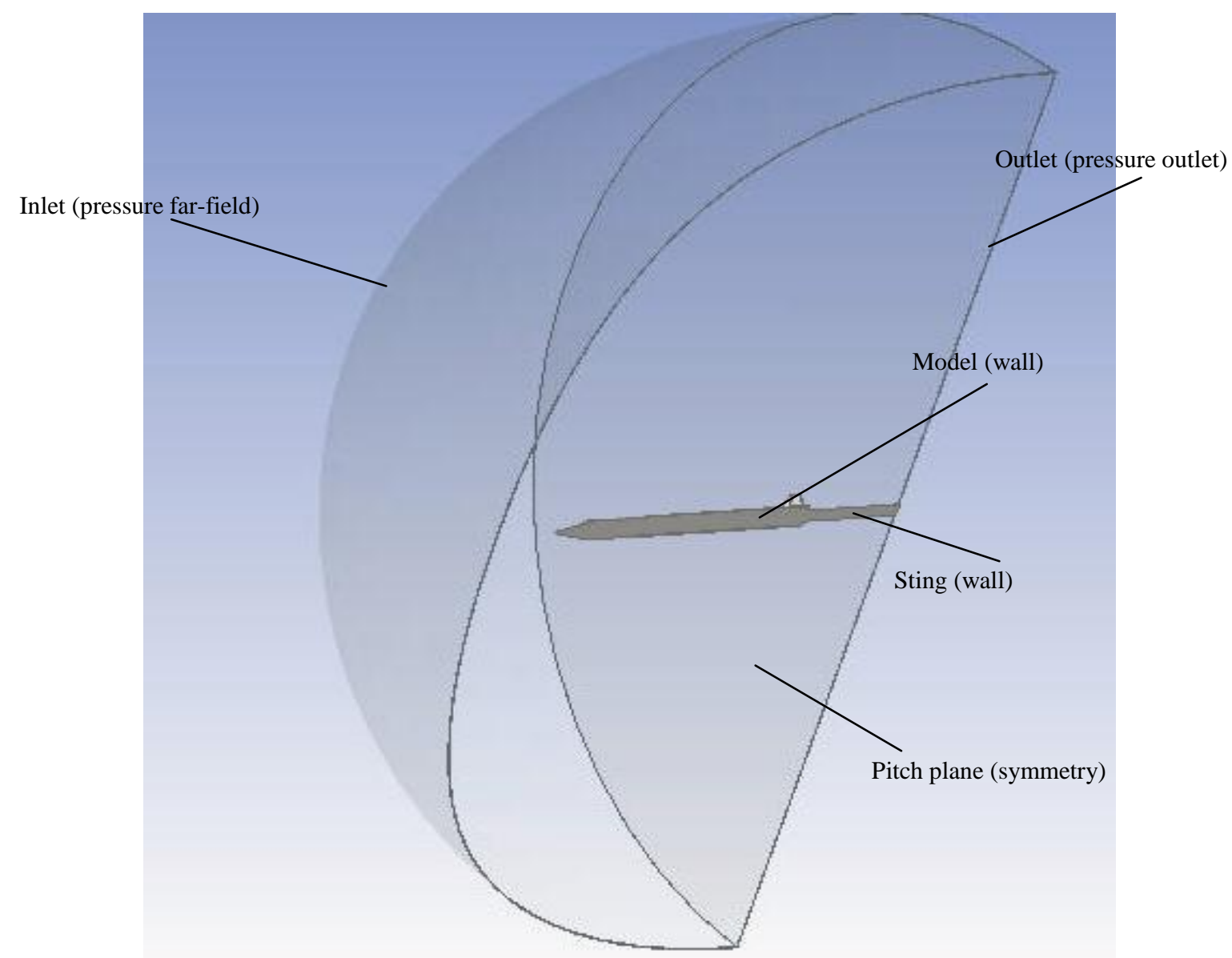

Fig. 4 Unbounded computational domain

\subsection{Domain discretization}

The two domains are then discretized. It's intended to generate grids that yield equal solution in both domains despite the difference in their configuration. the scoping methods available in the CFD solver[11] are thus adopted. Initially, a coarse unstructured mesh is generated in the domain. Then, the grid is refined at user-specified zones. These zones are chosen so as to enable resolving the key flow features. Two scoping methods are successively implemented; the element sizing then the sphere of influence sizing. Body element yields a clustered fine grid around the body whereas the sphere of influence is drawn in the areas of the domain where the shock and expansion waves take place. Five spheres of influence are drawn (marked by the thick circles in Fig. 5). Eventually, a nearly feature aligned grid is achieved. Fig. 5 shows the grid on the symmetry plane for both bounded and unbounded domains. 


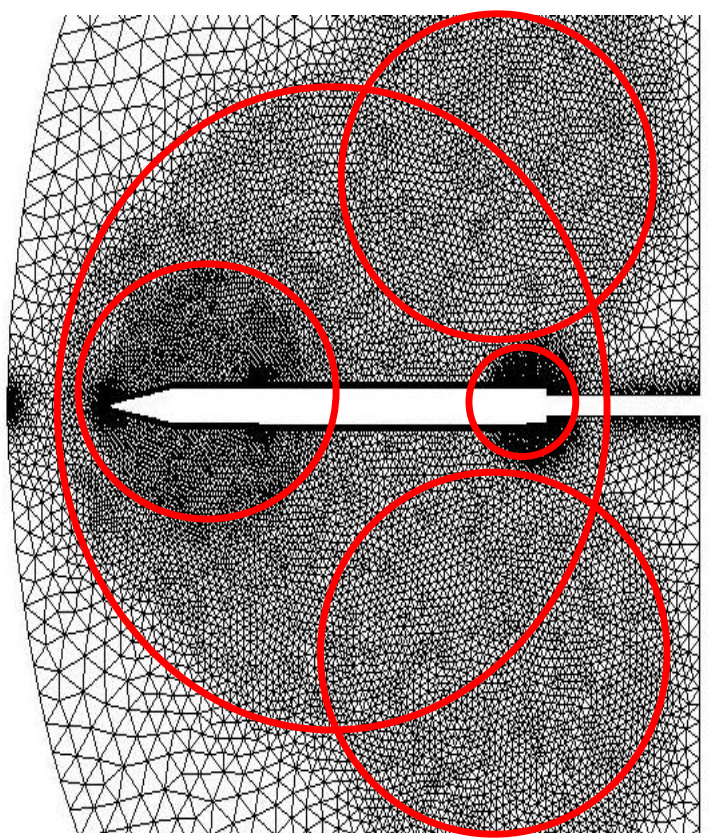

(a) Unbounded domain

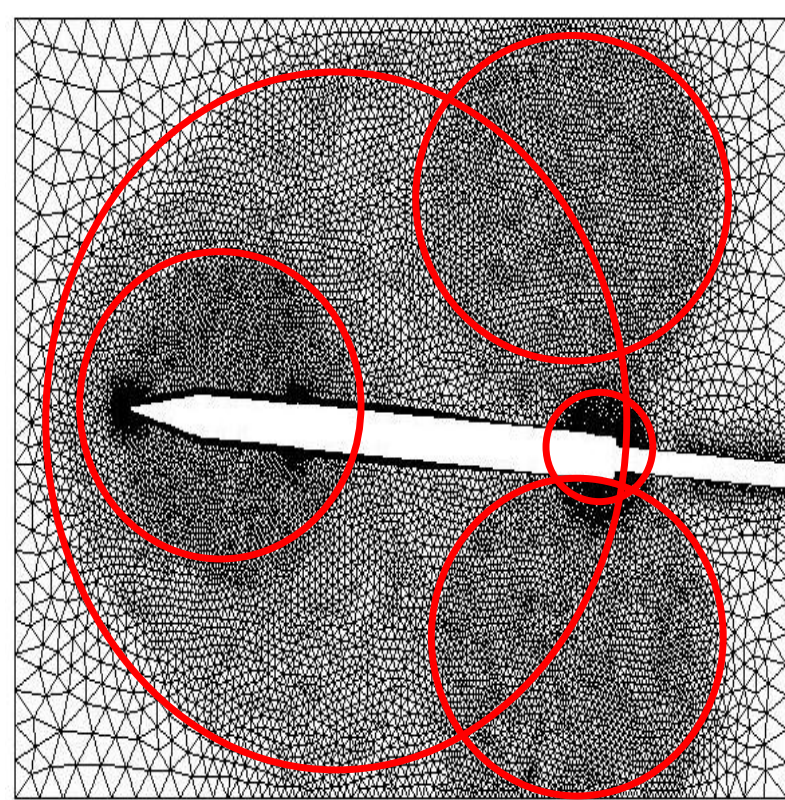

(b) Bounded domain

Fig. 5 Domain discretization using sphere of influence scoping method

A grid sensitivity check is then conducted to address how refining the grid improves the solution. This is achieved by continuously refining the grid and comparing the successive simulation results until no significant improvement is achieved. The solution is then said to be grid-independent solution.

Three flow simulations are run based on these grids. The features of these grids and the resulting fore drag coefficients are compared with the experimental data Table 2. The improvement between the second and the third grid is minor, so the coarser grid (the second grid) is chosen.

Table 2 Grid sensitivity check results

\begin{tabular}{l|l|l|l}
\hline Grid & No. of cells & $\mathrm{C}_{\mathrm{df}}$ & $\%$ improvement \\
\hline \hline 1 & 602061 & 0.376 & --------- \\
\hline 2 & 1825562 & 0.404 & 7.4 \\
\hline 3 & 2216425 & 0.4189 & 3.6 \\
\hline \hline
\end{tabular}

\subsection{Turbulence model}

The criterion for choosing the appropriate turbulence model for the present case is the nature of the flow. Spalart Almaras is designed specifically for applications where wall bounded flows are involved. [11].

\section{Results and discussion}

\section{1 features of the unbounded and bounded flow fields}

Mach contours and the stream lines along the symmetry plane for both bounded and unbounded domains at Mach 1.5, 2.5, and 4 are shown in Fig. 6 Contours and stream lines in both domains at different Mach numbers a, b, and c, respectively. Bounded domain is shown on the lift whereas unbounded domain is shown on the right. 

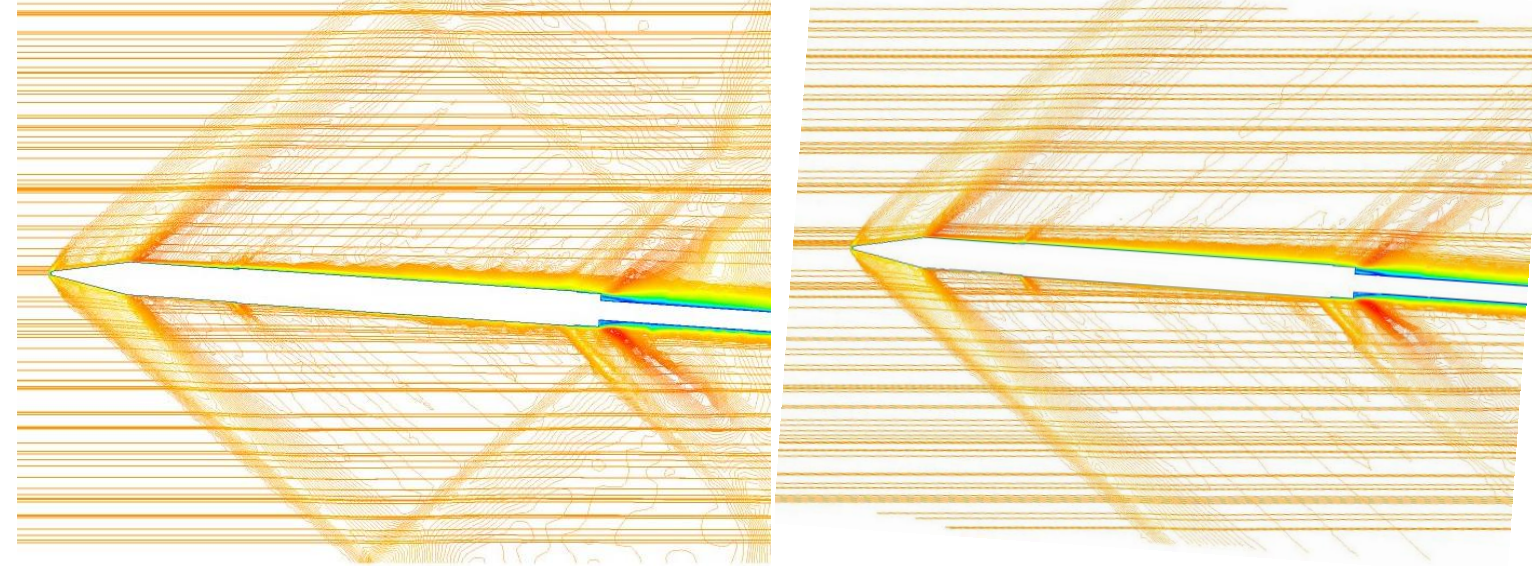

(a) $\mathrm{M}=1.5$
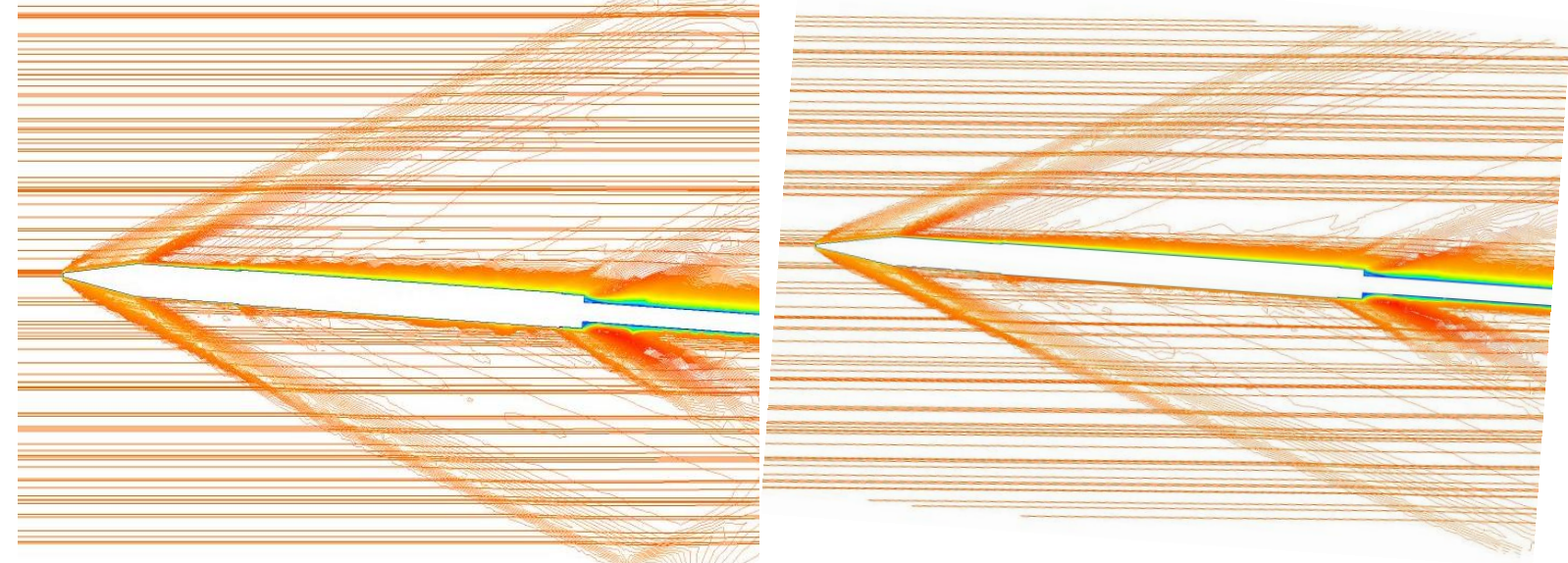

(b) $\mathrm{M}=2.5$
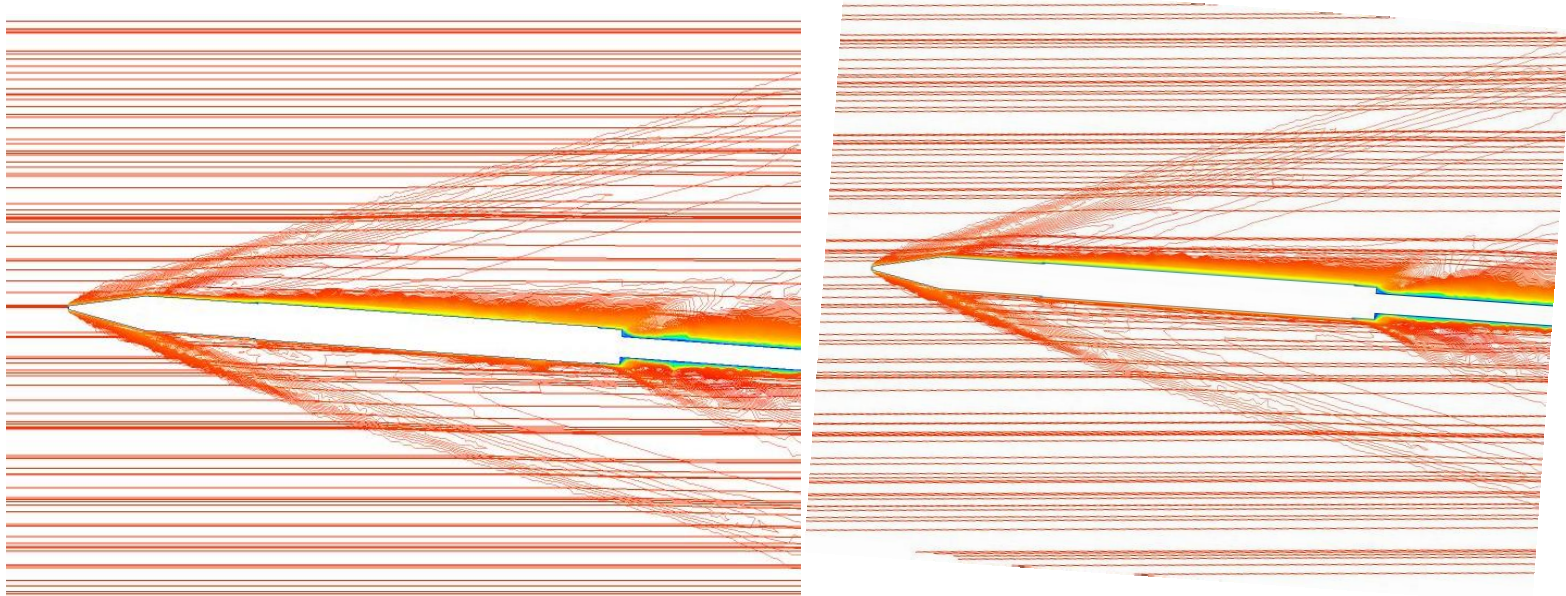

(c) $\mathrm{M}=4$

Fig. 6 Contours and stream lines in both domains at different Mach numbers

As shown in Fig. 6a, at Mach=1.5 a shock wave is created ahead of the model nose. The windward side of this conical shock is stronger that it's leeward side due to the incidence of the model. At the nose shoulder, expansion fan is created. At the beginning of the upper and lower ducts, an oblique shock is created. Another shock wave is created at the wing leading edge. At the base another expansion occurs ahead of the wake behind the model. In addition, the boundary layer around the model is thinner in the windward side than in the leeward side of the model. The wake is not fully developed due to the existence of the sting at the model base. 
As the Mach number increases to 2.5 in Fig. 6b, the shock wave ahead of the model nose begins to be more oblique and expansion at the nose shoulder is weaker than that of Mach 1.5. Finally at Mach 4 Fig. 6c, the shock ahead of the model nose becomes closer to the body wall and the oblique shocks at the ducts diminish especially at the upper duct. Moreover, expansion at the lower nose shoulder is clearer than that of the upper. Also, expansion at the lower base is not as significant as that at lower Mach.

In all Mach values, it seems that the curvature of streamlines around the model is confined in a small area away from the model. This can be addressed in both bounded and bounded domain. Away from the model and in the vicinity of the bounded domain boundaries, the streamlines are straight and are, hence, not affected by the presence of the tunnel walls. This may be owed to the nature of supersonic flow as well as the slenderness of the model.

At Mach 1.5 in the bounded domain, the shock generated ahead of the model nose impinges the upper and lower surfaces in the bounded domain. The reflected shock (which is stronger in the windward side) interferes with the model closer to the model fins and base area. As Mach increases to 2.5, reflection migrates downstream such that the reflected wave interferes with the model wave far downstream of the model base.

Pressure contours on the model surface Fig. 7 pressure contours on the model surfaceon the windward and leeward sides of the model in Mach 2.

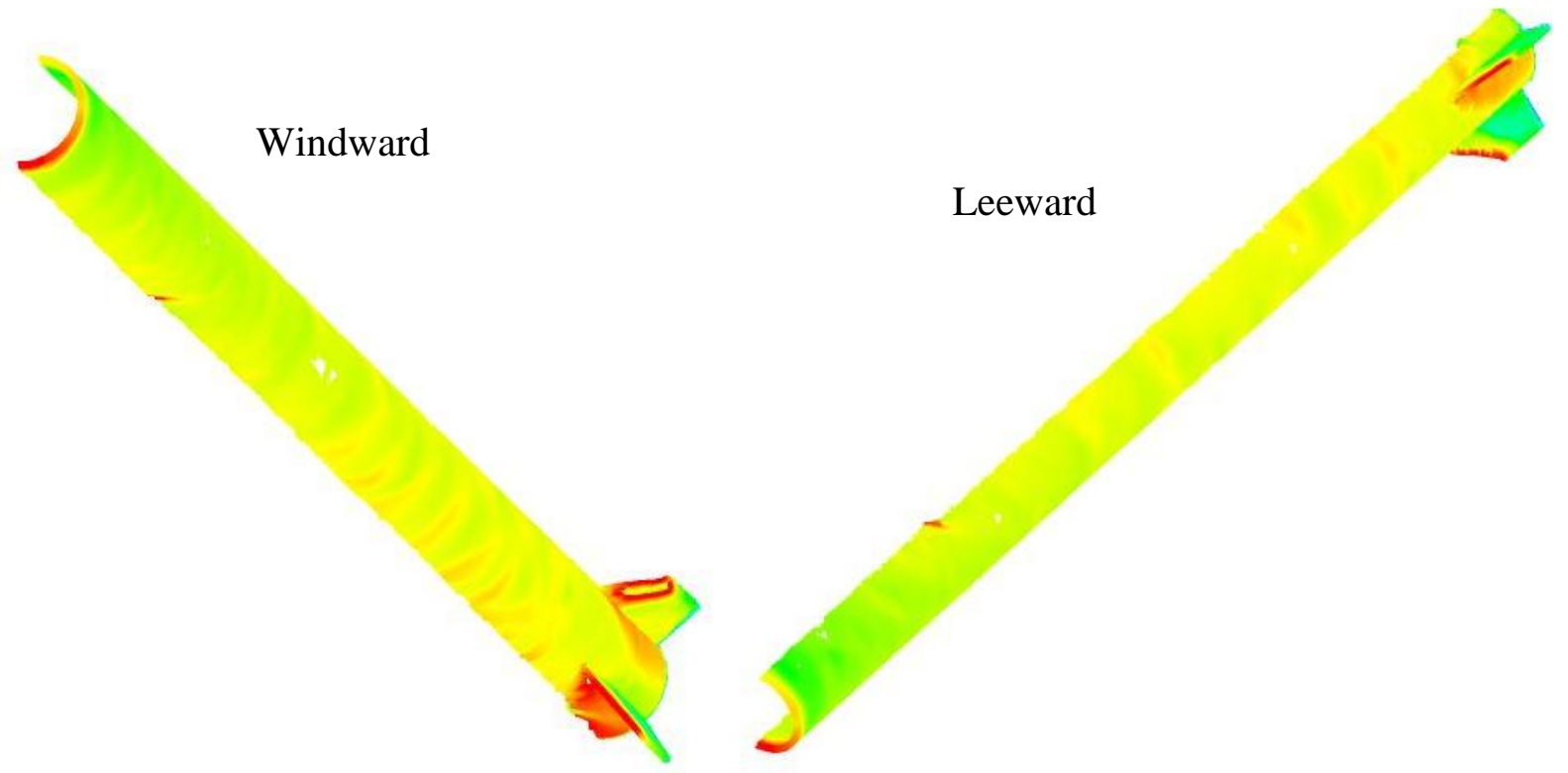

Fig. 7 pressure contours on the model surface

As shown in the above figure, the pressure at the nose tip is the highest value as it is nearly the stagnation pressure. The pressure on the lower side of the nose is much higher than that on the upper side that is the result for the incidence, the same feature for that at the lower an upper sides of the wing is shown in the figure. At the base, the pressure is the lowest value where the wake zone is generated.

\subsection{Variation of aerodynamic coefficients with Mach number}

\section{a. Normal force coefficient}

The change in the normal force coefficient with the Mach number is shown in Fig. 8 Change in normal force coefficient with the Mach number $\left(\alpha=4^{\circ}\right)$ as predicted by both bounded and unbounded solutions along with the experimental measurements. 


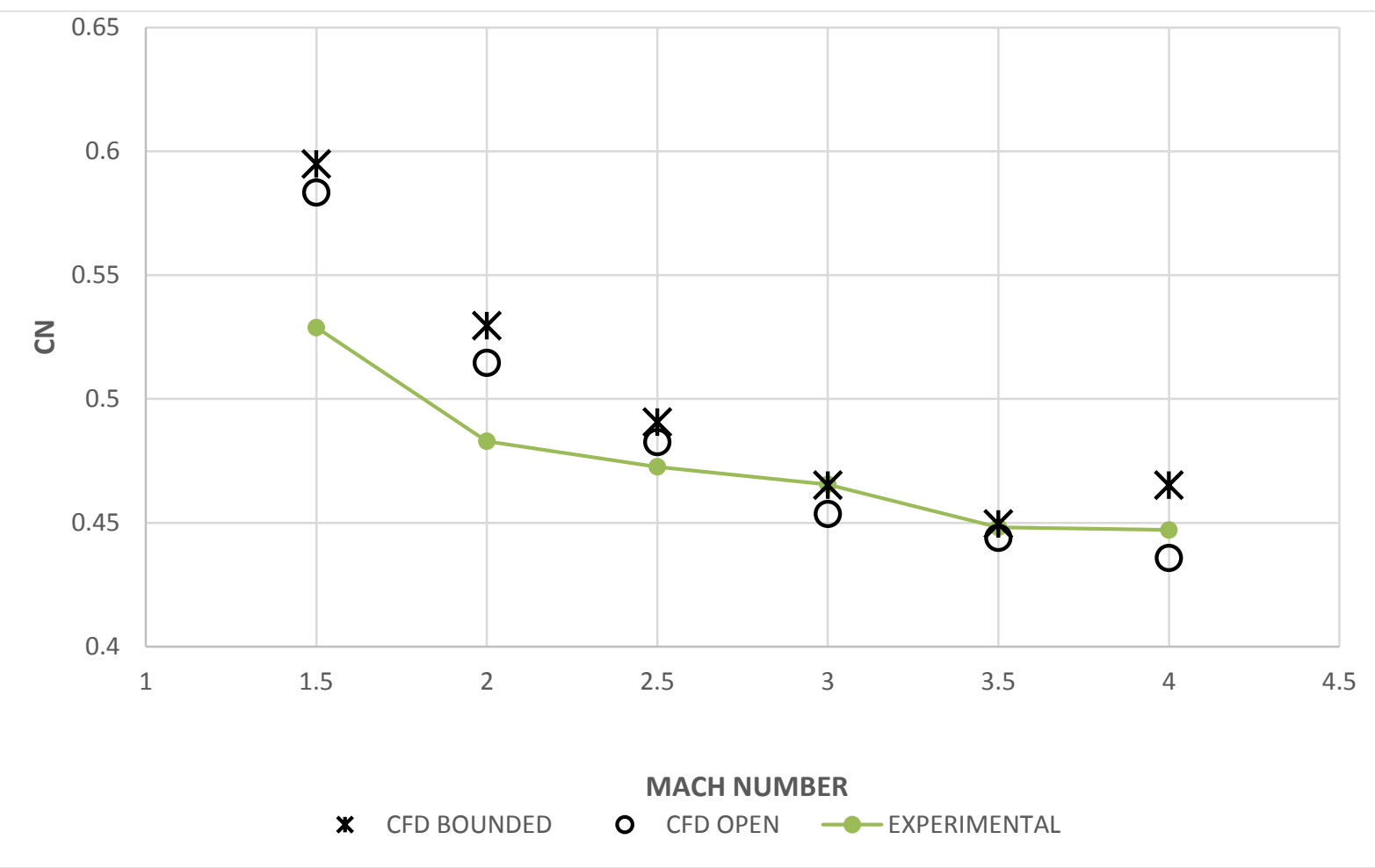

Fig. 8 Change in normal force coefficient with the Mach number $\left(\alpha=4^{\circ}\right)$

The measured normal force coefficient decreases as the Mach number increases. The simulation results based on both of the computational domains follows the same trend as the experimental ones, but with some difference of about $10 \%$ at Mach number 1.5, which is being currently investigated.

At higher Mach numbers, the values of the normal force coefficients based on both domains are almost equal to each other and to the measured values. This may indicate that the wind tunnel walls have insignificant effect on the measured model aerodynamic parameters at high Mach numbers.

b. Axial force coefficient

The change in axial force coefficient with the Mach number is shown in Fig. 9

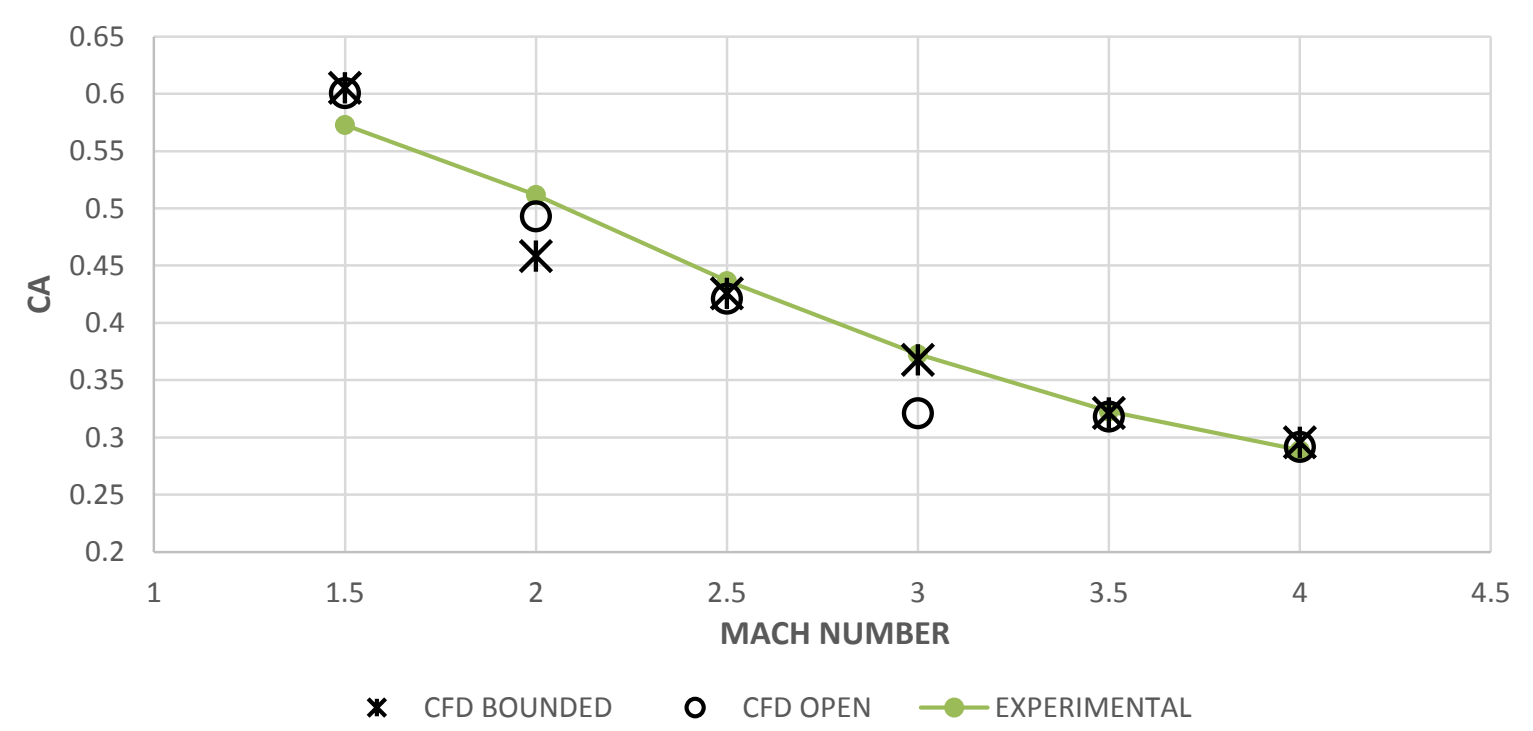

Fig. 9 Change in axial force coefficient with the Mach number 
The measured axial force coefficient decreases as the Mach number increases. Both of the two computational domains are following the same trend, which means that the axial force coefficient is not affected by the presence of the test section walls.

c. Normalized center of pressure position

The variation of the normalized center of pressure position measured from the tip of body nose with the Mach number is shown in Fig. 10.

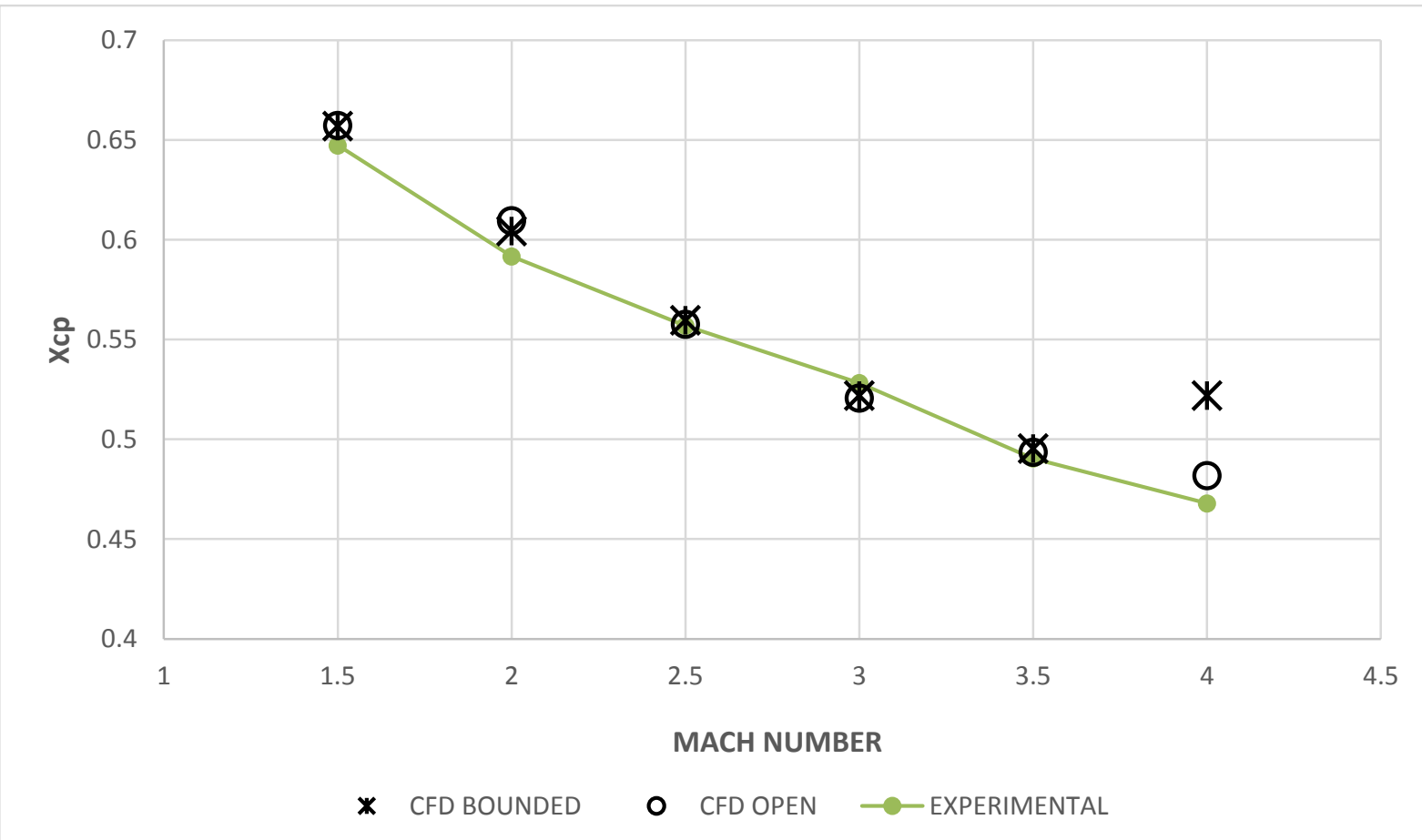

Fig. 10 change in normalized center of pressure with Mach number $\left(\alpha=4^{0}\right)$

The experimental data shows that the center of pressure shifts towards the body nose as the Mach number increases. The bounded computational domain shows the same behavior with an exception at $\mathrm{M}=4$. Similarly the unbounded computational domain results behavior agrees with that of the experimental ones. The definition of the simulation results at Mach 4 requires a deeper investigation.

\section{d. Drag polar}

To construct a drag polar based on simulation results, three additional simulations namely, at $10^{\circ}, 14^{\circ}$, and $18^{\circ}$ incidences, are conducted at every Mach number for the bounded domain. Fig. 11 shows the drag polar for Mach numbers 2 and 4. The drag polar points conducted from the computational domains, bounded or unbounded generally obey the trend of those conducted from experimental measurements 


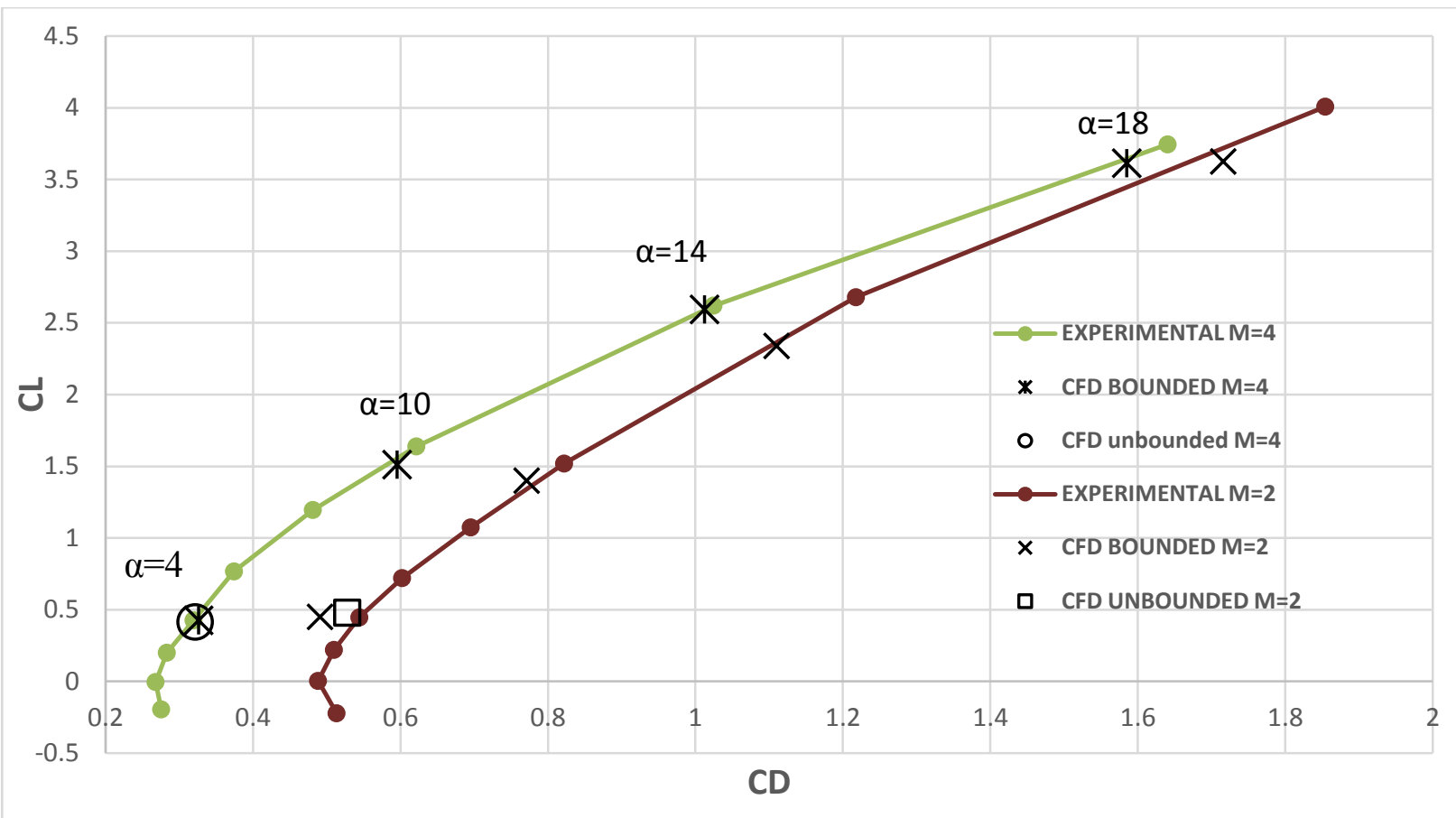

Fig. 11 Drag polar for Mach numbers 2 and 4

\section{Conclusion}

Numerical simulations are conducted to investigate the impact of the wind tunnel walls on the measured data in supersonic conditions. A model of a supersonic finned missile is taken as the case study and the free-stream conditions are taken corresponding to Mach 1.5 to 4 at $4^{\circ}$ incidence. The simulations are carried out using two three-dimensional computational domains, bounded and unbounded ones. Results from the simulations are mutually compared versus experimental measurements. Moreover, the flow field associated with both domains are analyzed to show the considered effects.

For the case considered, numerical simulations have shown a fairly accurate and reliable tool in predicting the aerodynamic coefficients, as compared with the experimental measurements. It was found that, the presence of wind tunnel walls has insignificant impacts on the measured data in the tunnel. However, at low supersonic Mach numbers, slight impact can be addressed possibly due to the shock reflections on the walls of the test section. This should be taken into consideration when correcting the tunnel data to resemble the real flight conditions.

The present study considered a fixed incidence angle and specific test section blockage. The study is currently extended to investigate the impact of test section blockage and incidence angle on the quality of the tunnel measurements and the associated flow field features.

\section{References}

[1] D. Althaus, "Tunnel-Wall Corrections at the Laminar Wind Tunnel," Institute report, IAG, http://www. iag. uni-stuttgart. de/laminarwindkanal, 2003.

[2] H. J. Allen and W. G. Vincenti, "Wall interference in a two-dimensional-flow wind tunnel, with consideration of the effect of compressibility," 1944.

[3] A. Thom and L. Klanfer, Tunnel-Wall Effect on an Aerofoil at Subsonic Speeds: HM Stationery Office, 1957.

[4] E. Maskell, "A theory of the blockage effects on bluff bodies and stalled wings in a closed wind tunnel," DTIC Document1963. 
[5] H. Awbi, "Wind-tunnel-wall constraint on two-dimensional rectangular-section prisms," Journal of Wind Engineering and Industrial Aerodynamics, vol. 3, pp. 285-306, 1978.

[6] G. S. West and C. J. Apelt, "The effects of tunnel blockage and aspect ratio on the mean flow past a circular cylinder with Reynolds numbers between 104 and 105," Journal of Fluid Mechanics, vol. 114, pp. 361-377, 1982.

[7] K. Duraisamy, W. J. McCroskey, and J. D. Baeder, "Analysis of wind tunnel wall interference effects on subsonic unsteady airfoil flows," Journal of aircraft, vol. 44, pp. 1683-1690, 2007.

[8] A. Ivanov and E. Streltsov, "Controlled Boundary Layer on the Solid Walls of Wind Tunnels: New Approach to The Boundary Interference Problem."

[9] G. Doig, T. J. Barber, E. Leonardi, A. J. Neely, and H. Kleine, "Aerodynamics of a supersonic projectile in proximity to a solid surface," AIAA journal, vol. 48, pp. 29162930, 2010.

[10] A. Roychowdhury, C. Unnikrishnan, and R. Aerodynamics, "Numerical Simulation of Wind tunnel Blockage and Wall Interference Effects at Supersonic Mach Number Flows," International Journal of Innovative Research in Technology \& Science(IJIRTS) vol. 2, pp. 63-72, 2013.

[11] I. ANSYS/FLUENT, "ANSYS/FLUENT, Inc. "Fluent 14.5 user guide,"", ed, 2014.

[12] DSSC Technicl Report TR70120, 2012. 